\title{
New Results on Boomerang and Rectangle Attacks ${ }^{\star}$
}

\author{
Eli Biham ${ }^{1}$, Orr Dunkelman ${ }^{1}$, and Nathan Keller ${ }^{2}$ \\ 1 Computer Science Department, Technion. \\ Haifa 32000, Israel \\ \{biham, orrd\}@cs.technion.ac.il \\ 2 Mathematics Department, Technion. \\ Haifa 32000, Israel \\ nkeller@tx.technion.ac.il
}

\begin{abstract}
The boomerang attack is a new and very powerful cryptanalytic technique. However, due to the adaptive chosen plaintext and ciphertext nature of the attack, boomerang key recovery attacks that retrieve key material on both sides of the boomerang distinguisher are hard to mount. We also present a method for using a boomerang distinguisher, which enables retrieving subkey bits on both sides of the boomerang distinguisher. The rectangle attack evolved from the boomerang attack.In this paper we present a new algorithm which improves the results of the rectangle attack.

Using these improvements we can attack 3.5-round SC2000 with $2^{67}$ adaptive chosen plaintexts and ciphertexts, and 10-round Serpent with time complexity of $2^{173.8}$ memory accesses (which are equivalent to $2^{165.3}$ Serpent encryptions) with data complexity of $2^{126.3}$ chosen plaintexts.
\end{abstract}

\section{Introduction}

Differential cryptanalysis 3 is based on studying the propagation of differences through an encryption function. Since its introduction many techniques based on it were introduced. Some of these techniques, like the truncated differentials [11] and the higher order differentials 2211, are generalizations of the differential attack. Some other techniques like differential-linear attack 14] and the boomerang attack [18] use the differential attack as a building block.

The boomerang attack is an adaptive chosen plaintext and ciphertext attack. It is based on a pair of short differential characteristics used in a specially built quartet. In the attack a pair of plaintexts with a given input difference are encrypted. Their ciphertexts are used to compute two other ciphertexts according to some other difference, these new ciphertexts are then decrypted, and the difference after the decryption is compared to some (fixed known) value.

\footnotetext{
* The work described in this paper has been supported by the European Commission through the IST Programme under Contract IST-1999-12324.
} 
The boomerang attack was further developed in [10] into a chosen plaintext attack called the amplified boomerang attack. Later, the amplified boomerang attack was further developed into the rectangle attack 7].

In the transition from the boomerang attack to the rectangle attack the probability of the distinguisher is reduced (in exchange for easing the requirements from adaptive chosen plaintext and ciphertext attack to a chosen plaintext attack). The reduction in the distinguisher's probability results in higher data complexity requirements. For example, the data requirements for distinguishing a 2.5-round SC2000 [17] from a random permutation using a rectangle distinguisher is $2^{84.6}$ chosen plaintext blocks, whereas only $2^{39.2}$ adaptive chosen plaintext and ciphertext blocks are required for the boomerang distinguisher.

In this paper we present a method to retrieve more subkey bits in key recovery boomerang attacks. We also present a better algorithm to perform rectangle attacks. These improvements result in better key recovery attacks which require less data or time (or both) and are more effective. The improvement to the generic rectangle attack reduces the time complexity of attacking 10-round Serpent from $2^{217}$ memory accesse 1 to $2^{173.8}$ memory accesses which are equivalent to about $2^{166.3}$ 10-round Serpent encryptions. We also prove that these key recovery attacks succeed (with very high probability) assuming that the distinguishers are successful.

The paper is organized as follows: In Section 2 we briefly describe the boomerang and the rectangle attacks. In Section 3 we present our new optimized generic rectangle attack and analyze its application to generic ciphers and to SC2000 and Serpent. In Section 4 we present our optimized generic boomerang attack and analyze its application to both a generic cipher and real blockciphers like SC2000 and Serpent. Section 5 describes a new technique to transform a boomerang distinguisher into a key recovery attack that retrieves more subkey material. We summarize this paper and our new results in Section 6.

\section{Introduction to Boomerang and Rectangle Attacks}

\subsection{The Boomerang Attack}

The boomerang attack was introduced in [18. The main idea behind the boomerang attack is to use two short differentials with high probabilities instead of one differential of more rounds with low probability. The motivation for such an attack is quite apparent, as it is easier to find short differentials with a high probability than finding a long one with a high enough probability.

We assume that a block cipher $E:\{0,1\}^{n} \times\{0,1\}^{k} \rightarrow\{0,1\}^{n}$ can be described as a cascade $E=E_{1} \circ E_{0}$, such that for $E_{0}$ there exists a differential $\alpha \rightarrow \beta$ with probability $p$, and for $E_{1}$ there exists a differential $\gamma \rightarrow \delta$ with probability $q$. The boomerang attack uses the first characteristic $(\alpha \rightarrow \beta)$ for $E_{0}$ with respect to the pairs $\left(P_{1}, P_{2}\right)$ and $\left(P_{3}, P_{4}\right)$, and uses the second characteristic

\footnotetext{
${ }^{1}$ In [7] it was claimed to be $2^{205}$ due to an error that occurred in the analysis.
} 
$(\gamma \rightarrow \delta)$ for $E_{1}$ with respect to the pairs $\left(C_{1}, C_{3}\right)$ and $\left(C_{2}, C_{4}\right)$. The attack is based on the following boomerang process:

- Ask for the encryption of a pair of plaintexts $\left(P_{1}, P_{2}\right)$ such that $P_{1} \oplus P_{2}=\alpha$ and denote the corresponding ciphertexts by $\left(C_{1}, C_{2}\right)$.

- Calculate $C_{3}=C_{1} \oplus \delta$ and $C_{4}=C_{2} \oplus \delta$, and ask for the decryption of the pair $\left(C_{3}, C_{4}\right)$. Denote the corresponding plaintexts by $\left(P_{3}, P_{4}\right)$.

- Check whether $P_{3} \oplus P_{4}=\alpha$.

We call these steps (encryption, XOR by $\delta$ and then decryption) a $\delta$-shift.

For a random permutation the probability that the last condition is satisfied is $2^{-n}$. For $E$, however, the probability that the pair $\left(P_{1}, P_{2}\right)$ is a right pair with respect to the first differential $(\alpha \rightarrow \beta)$ is $p$. The probability that both pairs $\left(C_{1}, C_{3}\right)$ and $\left(C_{2}, C_{4}\right)$ are right pairs with respect to the second differential is $q^{2}$. If all these are right pairs, then they satisfy $E_{1}^{-1}\left(C_{3}\right) \oplus E_{1}^{-1}\left(C_{4}\right)=\beta=E_{0}\left(P_{3}\right) \oplus$ $E_{0}\left(P_{4}\right)$, and thus, with probability $p$ also $P_{3} \oplus P_{4}=\alpha$. Therefore, the probability of this quartet of plaintexts and ciphertexts to satisfy the boomerang conditions is $(p q)^{2}$. Therefore, $p q>2^{-n / 2}$ must hold for the boomerang distinguisher (and the boomerang key recovery attacks) to work.

The attack can be mounted for all possible $\beta$ 's and $\gamma$ 's simultaneously (as long as $\beta \neq \gamma$ ), thus, a right quartet for $E$ is built with probability $(\hat{p} \hat{q})^{2}$, where:

$$
\hat{p}=\sqrt{\sum_{\substack{\beta \\ \alpha \rightarrow \beta}} \operatorname{Pr}^{2}[\alpha \rightarrow \beta]} \text { and } \hat{q}=\sqrt{\sum_{\substack{\gamma \rightarrow \delta \\ \gamma \rightarrow \delta}} \operatorname{Pr}^{2}[\gamma \rightarrow \delta]} .
$$

We refer the reader to [18,7] for the complete description and the analysis.

\subsection{The Rectangle Attack}

Converting adaptive chosen plaintext and ciphertext distinguishers into key recovery attacks pose several difficulties. Unlike the regular known plaintext, chosen plaintext, or chosen ciphertext distinguishers, using the regular methods of 3 3 15 114,5 14 to use adaptive chosen plaintext and ciphertext distinguishers in key recovery attacks fail, as these techniques require the ability to directly control either the input or the output of the encryption function.

In 10] the amplified boomerang attack is presented. This is a method for eliminating the need of adaptive chosen plaintexts and ciphertexts. The amplified boomerang attack achieves this goal by encrypting many pairs with input difference $\alpha$, and looking for a quartet (pair of pairs) for which, $C_{1} \oplus C_{3}=C_{2} \oplus C_{4}=\delta$ when $P_{1} \oplus P_{2}=P_{3} \oplus P_{4}=\alpha$. Given the same decomposition of $E$ as before, and the same basic differentials $\alpha \rightarrow \beta, \gamma \rightarrow \delta$, the analysis shows that the probability of a quartet to be a right quartet is $2^{-(n+1) / 2} p q$.

The reason for the lower probability is that no one can guarantee that the $\gamma$ difference (in the middle of the encryption; needed for the quartet to be a right boomerang quartet) is achieved. The lower probability makes the additional 
problem (already mentioned earlier) of finding and identifying the right quartets even more difficult.

The rectangle attack [7] shows that it is possible to count over all the possible $\beta$ 's and $\gamma$ 's, and presents additional improvements over the amplified boomerang attack. The improvements presented in the rectangle attack improve the probability of a quartet to be a right rectangle quartet to $\left.2^{-n / 2} \hat{p} \hat{q}\right|^{2}$

\section{Improving the Rectangle Attack}

The main problem dealt in previous works is the large number of possible quartets. Unlike in the boomerang attack, in which the identification of possible quartets is relatively simple, it is hard to find the right quartets in the rectangle attacks since the attacker encrypts a large number of pairs (or structures) and then has to find the right quartets through analysis of the ciphertexts. As the number of possible quartets is quadratic in the number of pair\$3, and as the attacker has to test all the quartets, it is evident that the time complexity of the attack is very large.

In this section we present an algorithm which solves the above problem by exploiting the properties of a right quartet, and which tests only a small part of the possible quartets. The new algorithm is presented on a generic cipher with the following parameters: Let $E$ be a cipher which can be described as a cascade $E=E_{f} \circ E_{1} \circ E_{0} \circ E_{b}$, and assume that $E_{0}$ and $E_{1}$ satisfy the properties of $E_{0}$ and $E_{1}$ presented in Section 2 (i.e., there exist differentials $\alpha \rightarrow \beta$ with probability $p$ of $E_{0}$ and $\gamma \rightarrow \delta$ with probability $q$ of $\left.E_{1}\right)$. An outline of such an $E$ is presented in Figure 1]. We can treat this $E$ as composed of $E^{\prime}=E_{1} \circ E_{0}$ (for which we have a distinguisher) surrounded by the additional rounds of $E_{b}$ and $E_{f}$. As mentioned in Section 2 , for sufficiently high probabilities $\hat{p}, \hat{q}$, we can distinguish $E_{1} \circ E_{0}$ from a random permutation using either a boomerang or a rectangle distinguisher. However, we also like to mount key recovery attacks on the full $E$.

Recall that the rectangle distinguisher parameters are $\alpha, \delta, \hat{p}$, and $\hat{q}$. Given these parameters, the rectangle distinguisher of the cipher $E^{\prime}=E_{1} \circ E_{0}$ can easily be constructed.

Before we continue we introduce some additional notations: Let $X_{b}$ be the set of all plaintext differences that may cause a difference $\alpha$ after $E_{b}$. Let $V_{b}$ be the space spanned by the values in $X_{b}$. Note that usually $n-r_{b}$ bits are set to 0 for all the values in $V_{b}$. Let $r_{b}=\log _{2}\left|V_{b}\right|$ and $t_{b}=\log _{2}\left|X_{b}\right|\left(r_{b}\right.$ and $t_{b}$ are not necessarily integers). Let $m_{b}$ be the number of subkey bits which enter $E_{b}$ and affect the difference of the plaintexts by decrypting pairs whose difference after $E_{b}$ is $\alpha$, or formally

\footnotetext{
${ }^{2}$ This is a lower bound for the probability. For further analysis see [7].

${ }^{3}$ In the rectangle attack the quartet $[(x, y),(z, w)]$ differs from the quartet $[(x, y),(w, z)]$.
} 


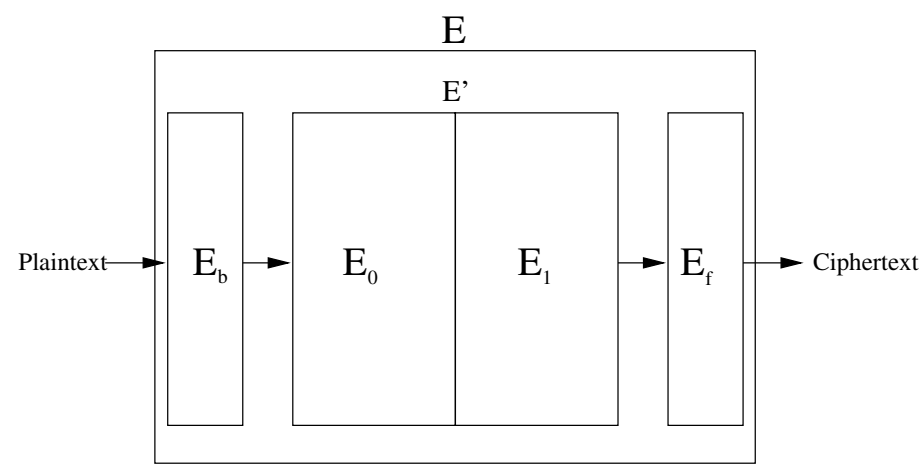

Fig. 1. Outline of $E$

$$
m_{b}=\mid\left\{K^{\prime} \mid w\left(K^{\prime}\right)=1 \text { and } \exists K, x: \begin{array}{l}
E_{b_{K}}^{-1}(x) \oplus E_{b_{K}}^{-1}(x \oplus \alpha) \neq \\
E_{b_{K \oplus K^{\prime}}^{-1}}(x) \oplus E_{b_{K \oplus K^{\prime}}}^{-1}(x \oplus \alpha)
\end{array}\right\} \mid
$$

where $w(x)$ denotes the hamming weight of $x$.

Similarly, let $X_{f}$ is the set of all ciphertext differences that a difference $\delta$ before $E_{f}$ may cause. Let $V_{f}$ denote the space spanned by the values of $X_{f}$ and denote $r_{f}=\log _{2}\left|V_{f}\right|$. Let $t_{f}=\log _{2}\left|X_{f}\right|$. Let $m_{f}$ be the number of subkey bits which enter $E_{f}$ and affect the difference when encrypting a pair with difference $\delta$ or formally

$$
m_{f}=\mid\left\{K^{\prime} \mid w\left(K^{\prime}\right)=1 \text { and } \exists K, x: \begin{array}{l}
E_{f_{K}}(x) \oplus E_{f_{K}}(x \oplus \alpha) \neq \\
E_{f_{K \oplus K^{\prime}}}(x) \oplus E_{f_{K \oplus K^{\prime}}}(x \oplus \alpha)
\end{array}\right\} \mid .
$$

We outline all these notations in Figure 2 .

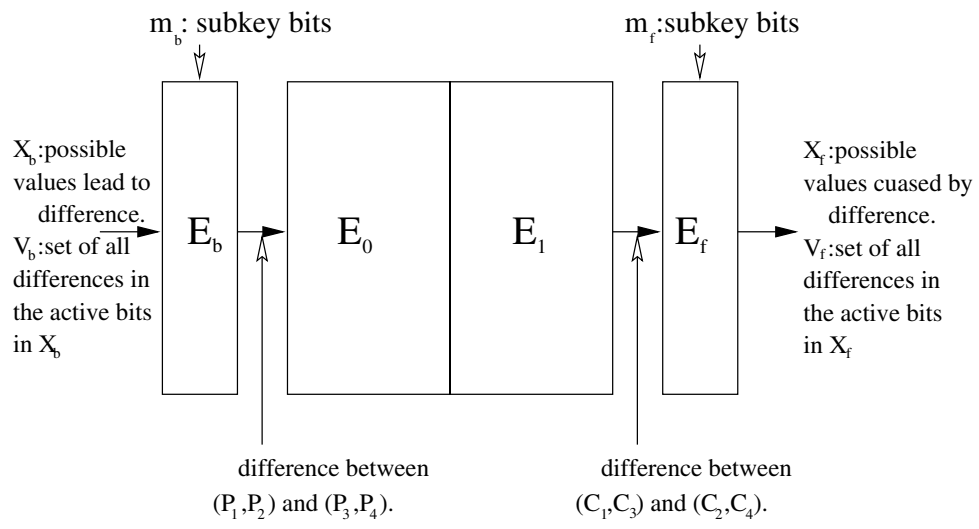

Fig. 2. The Notations Used in This Paper 
Our new algorithm for using rectangle distinguisher in a key recovery attack is as follows:

1. Create $Y=\left\lceil 2^{n / 2+2-r_{b}} / \hat{p} \hat{q}\right\rceil$ structures of $2^{r_{b}}$ plaintexts each. In each structure choose $P_{0}$ randomly and let $L=P_{0} \oplus V_{b}$ be the set of plaintexts in the structure.

2. Initialize an array of $2^{m_{b}+m_{f}}$ counters. Each counter corresponds to a different guess of the $m_{b}$ subkey bits of $E_{b}$ and the $m_{f}$ subkey bits of $E_{f}$.

3. Insert the $N=Y \cdot 2^{r_{b}}$ ciphertexts into a hash table according to the $n-r_{f}$ ciphertext bits that are set to 0 in $V_{f}$. If a pair agrees on these $n-r_{f}$ bits, check whether the ciphertext difference is in $X_{f}$.

4. For each collision $\left(C_{1}, C_{2}\right)$ which remains, denote $C_{i}$ 's structure by $S_{C_{i}}$ and attach to $C_{1}$ the index of $S_{C_{2}}$ and vice versa.

5. In each structure $S$ we search for two ciphertexts $C_{1}$ and $C_{2}$ which are attached to some other $S^{\prime}$. When we find such a pair we check that the $P_{1} \oplus P_{2}$ (the corresponding plaintexts) is in $X_{b}$, and check the same for the plaintexts which $P_{1}$ and $P_{2}$ are related to.

6. For all the quartets which passed the last test denote by $\left(P_{1}, P_{2}, P_{3}, P_{4}\right)$ the plaintexts of a quartet and by $\left(C_{1}, C_{2}, C_{3}, C_{4}\right)$ the corresponding ciphertexts. Increment the counters which correspond to all subkeys $K_{b}, K_{f}$ (actually their bits which affect the $\alpha$ and $\delta$ differences, respectively) for which $E_{b_{K_{b}}}\left(P_{1}\right) \oplus E_{b_{K_{b}}}\left(P_{2}\right)=E_{b_{K_{b}}}\left(P_{3}\right) \oplus E_{b_{K_{b}}}\left(P_{4}\right)=\alpha$ and $E_{f_{K_{f}}}^{-1}\left(C_{1}\right) \oplus$ $E_{f_{K_{f}}}^{-1}\left(C_{3}\right)=E_{f_{K_{f}}}^{-1}\left(C_{2}\right) \oplus E_{f_{K_{f}}}^{-1}\left(C_{4}\right)=\delta$.

7. Output the subkey with maximal number of hits.

The data complexity of the attack is $N=2^{r_{b}} Y=2^{r_{b}}\left\lceil 2^{n / 2+2-r_{b}} / \hat{p} \hat{q}\right\rceil$ chosen plaintexts. The time complexity of Step 1 (the data collection step) is $N$ encryptions. The time complexity of Step 2 is $2^{m_{b}+m_{f}}$ memory accesses in a trivial implementation and only one memory access using a more suitable data structures (like B-trees).

Step 3 requires $N$ memory accesses for the insertion of the ciphertexts into a hash table (indexed by the $n-r_{f}$ bits which are set to 0 in $V_{f}$ ). The number of colliding pairs is about $N^{2} \cdot 2^{r_{f}-n} / 2$ as there are $N$ plaintexts divided into $2^{n-r_{f}}$ bins (each bin correspond to a value of the $n-r_{f}$ bits). Note that we not necessarily use all the bins due to large memory requirements (i.e., we can hash only according the first 30 bits set to 0 in $V_{f}$ ). For each collision we check whether the difference of the ciphertexts of the colliding pair belongs to $X_{f}$. We keep all the $2^{t_{f}}$ values of $X_{f}$ in a hash table, and thus, the check requires one memory access for each colliding pair. Out of the $2^{r_{f}}$ possible differences for a colliding pair, only $2^{t_{f}}$ differences are in $X_{f}$ (i.e., can occur in a right quartet), and thus, about $N^{2} \cdot 2^{t_{f}-n-1}$ pairs remain. The time complexity of this step is $N+N^{2} \cdot 2^{r_{f}-n-1}$ memory accesses on average.

Step 4 requires one memory access for each pair which passes the filtering of Step 3. In a real implementation it is wiser to implement Step 4 as part of Step 3, but we separate these steps for the sake of simpler analysis. As there are 
$N^{2} \cdot 2^{t_{f}-n-1}$ such pairs, the time complexity of this step is on average $N^{2} \cdot 2^{t_{f}-n-1}$ memory accesses.

Step 5 implements a search for possible quartets. In a right quartet both $\left(P_{1}, P_{2}\right)$ and $\left(P_{3}, P_{4}\right)$ must satisfy that $E_{b}\left(P_{1}\right) \oplus E_{b}\left(P_{2}\right)=E_{b}\left(P_{3}\right) \oplus E_{b}\left(P_{4}\right)=\alpha$, and thus any right quartet must be combined from some $P_{1}, P_{2} \in S$ and $P_{3}, P_{4} \in \tilde{S}$ where $S$ and $\tilde{S}$ are two (not necessarily distinct) structures. Moreover, a right quartet satisfies that $E_{f}^{-1}\left(C_{1}\right) \oplus E_{f}^{-1}\left(C_{3}\right)=E_{f}^{-1}\left(C_{2}\right) \oplus E_{f}^{-1}\left(C_{4}\right)=\delta$, and thus $C_{1}$ is attached to $S_{C_{3}}$ and $C_{2}$ is attached to $S_{C_{4}}$ and as $P_{3}, P_{4}$ are from the same structure then $-S_{C_{3}}=S_{C_{4}}$. Therefore, in each structure $S$ we search for colliding attachments, i.e., pairs of ciphertexts in $S$ which are attached to the same (other) structure $\tilde{S}$. The $N^{2} \cdot 2^{t_{f}-n-1}$ attachments (colliding pairs) are distributed over $Y$ structures, and we get that approximately $\left(N \cdot 2^{t_{f}+r_{b}-n-1}\right)^{2} / Y$ possible quartets are suggested in each structure (where a quartet corresponds to a pair of plaintexts from some structure attached to the same structure). We implement the test in the same manner as in Step 3, i.e., keeping a hash table $H_{S}$ for each structure $S$ and inserting each ciphertext $C$ to $H_{S_{C}}$ according to the index of the structure attached to $C$. Denoting the plaintexts of the suggested quartet by $\left(P_{1}, P_{2}, P_{3}, P_{4}\right)$ and their corresponding ciphertexts by $\left(C_{1}, C_{2}, C_{3}, C_{4}\right)$, we first check that $P_{1} \oplus P_{2} \in X_{b}$. This test requires one memory access for each possible quartet. The probability that the value $P_{1} \oplus P_{2}$ is in $X_{b}$ is $2^{t_{b}-r_{b}}$. A quartet which fails this test can be discarded immediately. Therefore, out of the $N^{2} \cdot 2^{2 t_{f}+2 r_{b}-2 n-2}$ possible quartets only $N^{2} \cdot 2^{2 t_{f}+r_{b}+t_{b}-2 n-2}$ quartets remain. As stated before, this filtering requires one memory accesses for each candidate quartet, thus the algorithm requires $N^{2} \cdot 2^{2 t_{f}+2 r_{b}-2 n-2}$ memory accesses. We can discard more quartets by testing whether $P_{3} \oplus P_{4} \in X_{b}$. In total this step requires $N^{2} \cdot 2^{2 t_{f}+2 r_{b}-2 n-2} \cdot\left(1+2^{t_{b}-r_{b}}\right)$ memory accesses and about $N^{2} \cdot 2^{2 t_{f}+2 t_{b}-2 n-2}$ quartets remain after this step.

In Step 6 we try to deduce the right subkey from the remaining quartets. Recall that a right quartet satisfies $E_{b}\left(P_{1}\right) \oplus E_{b}\left(P_{2}\right)=\alpha=E_{b}\left(P_{3}\right) \oplus E_{b}\left(P_{4}\right)$. Both pairs are encrypted by the same subkey, hence, a right quartet must agree on $K_{b}$ (the $m_{b}$ subkey bits which enter $E_{b}$ and affect the output difference $\alpha$ ). There are $2^{t_{b}}$ possible input differences that lead to $\alpha$ difference after $E_{b}$, therefore, $2^{m_{b}-t_{b}}$ subkeys on average take one of these values into the difference $\alpha$. As each pair suggests $2^{m_{b}-t_{b}}$ subkeys, they agree on average on $\left(2^{m_{b}-t_{b}}\right)^{2} / 2\left(2^{m_{b}}\right)=2^{m_{b}-2 t_{b}-1}$ subkeys for $E_{b}$. We can find these options by keeping in a precomputed table either the possible values for any pair on its own, or for the whole quartet. Repeating the analysis for $E_{f},\left(C_{1}, C_{3}\right)$, and $\left(C_{2}, C_{4}\right)$ we get about $2^{m_{f}-2 t_{f}-1}$ subkeys suggestions from each quartet. Thus, each of the remaining quartets suggests $2^{m_{b}+m_{f}-2 t_{f}-2 t_{b}-2}$ possible subkeys. There are $2^{m_{b}+m_{f}}$ possible subkeys and $N^{2} \cdot 2^{2 t_{f}+2 t_{b}-2 n-2} \cdot 2^{m_{b}+m_{f}-2 t_{f}-2_{b}-2}=N^{2} \cdot 2^{m_{b}+m_{f}-2 n-4}$ hits. The expected number of hits for a (wrong) subkey is about $N^{2} \cdot 2^{-2 n-4}$. Since $N \leq 2^{n}$ is the number of plaintexts the expected number of hits per wrong subkey is less than $2^{-4}=1 / 16$, and we can conclude that the attack almost always succeeds in recovering subkey bits (since the number of expected hits for the right subkey is 4 ), or at least reduces the number of candidates for the right subkey. We 
can insert the $2^{m_{b}-t_{b}}$ subkeys suggested by $\left(P_{1}, P_{2}\right)$ into a hash table, and for each subkey suggested by the pair $\left(P_{3}, P_{4}\right)$ we can check whether it was already suggested. Doing the same for $E_{f}$ we get that for each quartet we need 3 . $2^{m_{b}-t_{b}}+3 \cdot 2^{m_{f}-t_{f}}$ memory accesses. We can optimize this a little bit by storing in advance a table and a list for each difference in $X_{b}$ and save the time of building the hash table. This method saves $1 / 3$ of the number of memory accesses. Using this method this step requires about $N^{2} \cdot 2^{2 t_{f}+2 t_{b}-2 n-3} \cdot\left(2^{m_{b}-2 t_{b}}-2^{m_{f}-2 t_{f}}\right)=$ $N^{2} \cdot 2^{-2 n-3} \cdot\left(2^{m_{b}+2 t_{f}}+2^{m_{f}+2 t_{b}}\right)$ memory accesses for the entire attack.

Step 7 requires $2^{m_{b}+m_{f}}$ memory accesses using a trivial implementation, which can be reduced to 1-4 memory accesses using a more efficient data structure (e.g., B-trees or dynamic hash tables).

Overall, this algorithm requires $N=2^{r_{b}} Y=2^{r_{b}}\left\lceil 2^{n / 2+2-r_{b}} / \hat{p} \hat{q}\right\rceil$ chosen plaintexts, and time complexity of $N+N^{2}\left(2^{r_{f}-n-1}+2^{t_{f}-n}+2^{2 t_{f}+2 r_{b}-2 n-2}+\right.$ $\left.2^{m_{b}+t_{b}+2 t_{f}-2 n-1}+2^{m_{f}+2 t_{b}+t_{f}-2 n-1}\right)$ memory accesses. The memory complexity is $N+2^{t_{b}}+2^{t_{f}}+2^{m_{b}+m_{f}}$.

Table 1 summarizes the time complexity of each step and the number of plaintexts / pairs / quartets that remain after each step of the algorithm.

Table 1. The Rectangle Attack Steps and their Effect

\begin{tabular}{llll}
\hline Step Short & Time & Complexity & \# Remaining Texts/ \\
No. & Description & Pairs/Quartets \\
\hline 1 & Data generation & $N$ encryptions & N plaintexts \\
2 & Subkey counters' init. & $1 \mathrm{MA}$ & No change \\
3 & First filtering & $N+N^{2} 2^{r_{f}-n-1} \mathrm{MA}$ & $N^{2} \cdot 2^{t_{f}-n-1}$ pairs \\
4 & Suggesting quartets & $N^{2} 2^{t_{f}-n-1} \mathrm{MA}$ & No change \\
5 & Eliminating quartets & $N^{2} 2^{2 t_{f}+2 r_{b}-2 n-2} \mathrm{MA}$ & $N^{2} 2^{2 t_{f}+2 t_{b}-2 n-2}$ qts. \\
6 & Subkey detection & $N^{2} 2^{t_{b}+t_{f}-2 n-1}\left(2^{m_{b}+t_{f}}+2^{m_{f}+t_{b}}\right)$ \\
& & \multicolumn{2}{c}{ NA change } \\
7 & Printing subkey & $1-4 \mathrm{MA}$ & No change \\
\hline
\end{tabular}

MA - Memory Accesses

Using this algorithm we can break 3.5-round SC2000, using the following decomposition: $E_{b}$ consists of the first $\mathrm{S} 4$ layer, the following 1.25 rounds are $E_{0}$, the next 1.25 rounds are $E_{1}$, and the final $\mathrm{S} 4$ layer is $E_{f}$. For this decomposition the following properties were presented in [8]: $r_{b}=r_{f}=m_{b}=m_{f}=40, t_{b}=$ $27, t_{f}=27.9, n=128, \hat{p}=2^{-8.96}, \hat{q}=2^{-9.16}$. Thus, we conclude that the data complexity is $N=2^{84.6}$ chosen plaintexts and that the time complexity is $2^{84.6}$ memory accesses, which slightly improves the results in [8].

We can also break 10-round Serpent using the following decomposition: $E_{b}$ consists of round 0 . The following 4 rounds are $E_{0}$, the next 4 rounds are $E_{1}$, and round 9 is $E_{f}$. For this decomposition the following properties are presented in [7] $r_{b}=m_{b}=76, r_{f}=m_{f}=20, t_{b}=48.85, t_{f}=13.6, n=128, \hat{p}=$

${ }^{4}$ As stated earlier, in [7] it was mistakenly claimed that $t_{b}=64$. We use the correct value of 76 , and derive the correct time complexity. 
$2^{-25.4}, \hat{q}=2^{-34.9}$. Thus, we conclude that the data complexity is $N=2^{126.3}$ chosen plaintexts and that the time complexity is $2^{173.8}$ memory accesses. Note that the time complexity of the attack presented in [7] is $2^{217}$ memory accesses using $2^{196}$ memory cells (or $2^{219.4}$ memory accesses with $2^{131.8}$ memory cells).

Note that we can use the above algorithm in several other scenarios. One of them is a chosen ciphertext scenario, where the above algorithm is applied on $E^{-1}=E_{b}^{-1} \circ E_{0}^{-1} \circ E_{1}^{-1} \circ E_{f}^{-1}$. The analysis of this case is the same as before as long as we replace in the above equations all the sub-scripts $b$ by $f$ and vice versa.

Sometimes, we might want to attack a cipher $E$ with additional rounds only at one side, i.e., to divide $E$ to $E=E_{1} \circ E_{0} \circ E_{b}$ (or $E=E_{f} \circ E_{1} \circ E_{b}$ ). In this case our analysis still holds with $m_{f}=r_{f}=t_{f}=0$.

\section{A Key Recovery Attack Based on Boomerang Distinguisher}

In this section we apply our ideas from the previous section to the boomerang attack. We generalized the results of [18, . Like the rectangle attack, we have found that whenever the boomerang distinguisher succeeds then the key recovery attack also succeeds.

There are various standard techniques to use distinguishers for a key recovery attack [3,15,11,4,5]. The basic idea is to try all subkeys which affect the difference (or the approximation) before and after the distinguishers (i.e., in $E_{b}$ and $E_{f}$ ), and to deduce that the correct subkey is the one for which the statistical distinguisher has the best results. However, this basic idea can be very expensive in terms of time and memory complexities. Moreover, due to the adaptive chosen plaintext and ciphertext requirement, using a boomerang distinguisher in a key recovery attack can be done if either $E_{b}$ or $E_{f}$ is present but not when both exist.

As both boomerang and rectangle distinguishers exploit the same $\alpha$ and $\delta$, we use the same notations of $E=E_{f} \circ E_{1} \circ E_{0} \circ E_{b}, m_{b}, r_{b}, t_{b}, m_{f}, r_{f}$, and $t_{f}$ as in the earlier sections.

The generic boomerang attack on $E=E_{1} \circ E_{0} \circ E_{b}$ is as follows:

1. Initialize an array of $2^{m_{b}}$ counters. Each counter corresponds to a different guess of the $m_{b}$ subkey bits of $E_{b}$.

2. Generate a structure $F$ of plaintexts, choose $P_{0}$ randomly and let $F=P_{0} \oplus V_{b}$ be the set of plaintexts in the structure.

3. Ask for the encryption of $F$ and denote the set of ciphertexts by $G$.

4. For each ciphertext $c \in G$ compute $c^{\prime}=c \oplus \delta$, and define the set $H=$ $\{c \oplus \delta \mid c \in G\}$.

5. Ask for the decryption of $H$, and denote the plaintexts set by $I$.

6. Insert all the plaintexts in $I$ into a hash table according to the $n-r_{b}$ plaintext bits which are set to 0 in $V_{b}$.

7. In case of a collision of plaintexts in the hash table: 
a) Denote the plaintexts which collide in the hash table by $\left(P_{3}, P_{4}\right)$, and test whether $P_{3} \oplus P_{4} \in X_{b}$. If this condition is satisfied denote the plaintexts from $F$ which correspond to $\left(P_{3}, P_{4}\right)$ by $\left(P_{1}, P_{2}\right)$. Test whether $P_{1} \oplus P_{2} \in X_{b}$. If any of the tests fails, discard this quartet.

b) For a quartet $\left(P_{1}, P_{2}, P_{3}, P_{4}\right)$ which passes the above filtering we check all possible $K_{b}$ which enter $E_{b}$ (actually its bits which affect the $\alpha$ ) and increment the counters which correspond to the subkeys for which $E_{b_{K_{b}}}\left(P_{1}\right) \oplus E_{b_{K_{b}}}\left(P_{2}\right)=E_{b_{K_{b}}}\left(P_{3}\right) \oplus E_{b_{K_{b}}}\left(P_{4}\right)=\alpha$.

8. Repeat Steps $2-7$ until a subkey is suggested 4 times.

Steps 2-5 perform a $\delta$-shift on structures (and not on the pairs directly).

From the analysis in [18 and the properties of the algorithm it is evident that the data complexity of the attack is about $8(\hat{p} \hat{q})^{-2}$. However, we generate at least $2^{r_{b}+1}$ plaintexts and ciphertexts, and thus the data complexity of the attack is $N=\max \left\{2^{r_{b}+1}, 8(\hat{p} \hat{q})^{-2}\right\}$.

The time complexity of Step 1 is equivalent to $2^{m_{b}}$ memory accesses. However, we can keep the counters in more efficient data structures (like B-trees, or dynamic hash tables) for which Step 1 takes only one memory access.

In steps $2-5$ we encrypt $N / 2$ plaintexts, compute $N / 2$ XOR operations and decrypt $N / 2$ ciphertexts. Thus, the total time complexity of Steps $2-5$ for the whole attack is $N$ encryptions/decryptions.

Repeating the analysis from the previous section, we restrict our attention to time complexity analysis for each $F$ independently of other $F$ 's, as each execution of Steps 6-7 for a given structure is independent of the execution of these steps for other structures.

As we insert the plaintexts into a hash tables, each plaintext in $I$ requires one memory access. Thus, the time complexity of Step 6 is $2^{r_{b}}$ memory accesses per structure, and therefore $N / 2$ memory accesses for the entire attack.

Repeating the analysis from the previous section (with the relevant minor changes), we get that Step 7 for each structure $I$ (and therefore, for each $F$ ) requires about $2^{3 r_{b}-n-1}$ memory accesses. For the entire $N / 2^{r_{b}+1}$ structures this step requires $2^{3 r_{b}-n-1} \cdot N / 2^{r_{b}+1}=N \cdot 2^{2 r_{b}-n-1}$ memory accesses.

Using the same arguing about right quartets (as in right quartet both $\left(P_{1}, P_{2}\right)$ and $\left(P_{3}, P_{4}\right)$ have an $\alpha$ difference after $\left.E_{b}\right)$, we get that each pair suggests $2^{m_{b}-t_{b}}$ subkeys, and both pairs agree on $\left(2^{m_{b}-t_{b}}\right)^{2} / 2\left(2^{m_{b}}\right)=2^{m_{b}-2 t_{b}-1}$ subkeys for $E_{b}$ on average. Hence, the expected number of memory accesses in Step 7(b) is $2^{t_{b}+m_{b}+r_{b}-n}$ for each structure. We conclude that the total time complexity of Step 7(b) is expected to be $N \cdot 2^{t_{b}+m_{b}-n-1}$ memory accesses for the whole attack.

Each structure $F$ induces about $2^{2 t_{b}+r_{b}-n-1} \cdot 2^{m_{b}-2 t_{b}-1}=2^{r_{b}+m_{b}-n-2}$ subkey hits. As there are $N / 2^{r_{b}+1}$ structures, the total number of subkey hits is expected to be $N \cdot 2^{m_{b}-n-3}$, which are distributed over $2^{m_{b}}$ subkeys. Thus, the expected number of hits for each subkey is $N \cdot 2^{-n-3}$. As $N \leq 2^{n}$, the expected number of hits for a wrong subkey is less then $1 / 8$ while the right subkey is expected to get 4 hits. This is sufficient for either recovering the right subkey, or to reduce the subkey candidates space by a very large factor. 
In Step 8 we check whether one of the counters has the value of 4 (or more). This has to be done whenever we finish Step 7(b) for some $F$. We can implement this step as part of Step 7(b). Whenever a counter is increased we check that it has not exceeded 4. However, this method results in enlarging the time of Step 7(b). Using more appropriate data structures, we can perform the check once whenever we replace the $F$ structure we work with. This yields a time complexity of $N / 2^{r_{b}+1}$ memory accesses.

We conclude that the attack requires $N=\max \left\{2^{r_{b}+1}, 8(\hat{p} \hat{q})^{-2}\right\}$ adaptive chosen plaintexts and ciphertexts and time complexity of about $N\left(1+2^{2 r_{b}-n-2}+\right.$ $2^{t_{b}+m_{b}-n-1}$ ) memory accesses.

Table 2 summarizes the time complexity of each step and the number of plaintexts / pairs / quartets that remain after each step of the algorithm.

Table 2. The Basic Boomerang Attack Steps and their Effect

\begin{tabular}{llll}
\hline Step Short & Time & \# of Remaining Plaintexts/ \\
No. & Description & Complexity & Pairs/ Quartets \\
\hline 1 & Subkey counters' init. & $1 \mathrm{MA}$ & - \\
$2+3$ & Data generation & $N / 2$ encryptions & $N / 2$ plaintexts \\
4 & Data generation & $N / 2 \mathrm{MA}$ & No change \\
5 & Data generation & $N / 2$ decryptions & $N$ plaintexts \\
6 & Finding possible quartets $N / 2 \mathrm{MA}$ & $N \cdot 2^{2 r_{b}-n-2}$ quartets \\
$7($ a) & Eliminating quartets & $N \cdot 2^{2 r_{b}-n-2} \mathrm{MA}$ & $N \cdot 2^{2 t_{b}-n-2}$ quartets \\
$7($ b) & Subkey detection & $N \cdot 2^{r_{b}+m_{b}-n-1} \mathrm{MA}$ No change \\
8 & Printing subkey & $N / 2^{r_{b}+1} \mathrm{MA}$ & No change \\
\hline MA & Memory Accesses & \multicolumn{2}{l}{}
\end{tabular}

Using this algorithm, we can break 3-round SC2000, using the following decomposition: $E_{b}$ consists of the first $\mathrm{S} 4$ layer, the following 1.25 rounds are $E_{0}$ and the next 1.25 rounds are $E_{1}$. For this decomposition the following properties were presented in [8]: $r_{b}=m_{b}=40, t_{b}=27, n=128, \hat{p}=2^{-8.96}, \hat{q}=2^{-9.16}$. Thus, we conclude that the data complexity of the attack is $N=2^{41}$ adaptive chosen plaintexts and ciphertexts. The time complexity of the attack is about $2^{41}$ memory accesses.

We attack 9-round Serpent, using this algorithm and the following decomposition: $E_{b}$ consists of round 0 , the following 4 rounds are $E_{0}$, and the next 4 rounds are $E_{1}$. For this decomposition the following properties were presented in [7]: $r_{b}=m_{b}=76, t_{b}=48.85, n=128, \hat{p}=2^{-25.4}, \hat{q}=2^{-34.9}$. The data complexity of the attack is $N=2^{123.6}$ adaptive chosen plaintexts and ciphertexts, with time complexity of $2^{147.2}$ memory accesses. We can also attack rounds 1-9 of Serpent using the decomposition used in the previous section. In this attack we are attacking rounds $9-1$ (i.e., the attack is on $E_{0}^{-1} \circ E_{1}^{-1} \circ E_{f}^{-1}$ ). For this decomposition we get: $r_{f}=m_{f}=20, t_{f}=13.6, n=128, \hat{p}=2^{-25.4}, \hat{q}=2^{-34.9}$. Obviously the data complexity does not change, as we use the same underly- 
ing distinguisher. However, the time complexity of this attack drops to $2^{123.6}$ memory accesses (instead of $2^{147.2}$ ).

\section{Enhancing the Boomerang Attack}

In this section we present a new method to use the boomerang attack with both $E_{b}$ and $E_{f}$. Recall that the main step of the boomerang attack is the $\delta$-shift (encryption of each plaintext, XORing of the corresponding ciphertext with $\delta$, and decryption of the outcome). Our method uses a generalization of the $\delta$-shift.

The new algorithm to attack $E_{f} \circ E_{1} \circ E_{0} \circ E_{b}$ is as follows:

1. Initialize an array of $2^{m_{b}+m_{f}}$ counters. Each counter corresponds to a different guess of the $m_{b}$ subkey bits of $E_{b}$ and the $m_{f}$ bits of $E_{f}$.

2. Generate a structure $F$ of plaintexts, choose $P_{0}$ randomly and let $F=P_{0} \oplus V_{b}$ be the set of plaintexts in the structure.

3. Ask for the encryption of $F$ and denote the set of ciphertexts by $G$.

4. For each $c \in G$ and $\epsilon \in X_{f}$ compute $c^{\prime}=c \oplus \epsilon$, and define the set $H=$ $\left\{c \oplus \epsilon \mid c \in G\right.$ and $\left.\epsilon \in X_{f}\right\}$.

5. Ask for the decryption of $H$, and denote the plaintexts set by $I$.

6. Insert all the plaintexts in $I$ into a hash table according to the $n-r_{b}$ plaintext bits which are set to 0 in $V_{b}$.

7. In case of a collision of plaintexts in the hash table:

a) Denote the plaintexts which collide in the hash table by $\left(P_{3}, P_{4}\right)$, and test whether $P_{3} \oplus P_{4} \in X_{b}$. If this condition is satisfied denote the plaintexts from $F$ which correspond to $\left(P_{3}, P_{4}\right)$ by $\left(P_{1}, P_{2}\right)$. Test whether $P_{1} \oplus P_{2} \in X_{b}$. If any of the tests fails, discard this quartet.

b) For a quartet $\left(P_{1}, P_{2}, P_{3}, P_{4}\right)$ which passes the above filtering we obtain from a precomputed table the possible values for the $m_{b}$ subkey bits which enter $E_{b}$ and affect the $\alpha$ difference. We also obtain from a precomputed table the possible values for the $m_{f}$ subkey bits which enter $E_{f}$ and affect the $\delta$ difference. The specific implementation aspects of this step are described later on. We increment counters which correspond to subkeys $K_{b}, K_{f}$ for which $E_{b_{K_{b}}}\left(P_{1}\right) \oplus E_{b_{K_{b}}}\left(P_{2}\right)=E_{b_{K_{b}}}\left(P_{3}\right) \oplus E_{b_{K_{b}}}\left(P_{4}\right)=\alpha$ and $E_{f_{K_{f}}}^{-1}\left(C_{1}\right) \oplus E_{f_{K_{f}}}^{-1}\left(C_{3}\right)=E_{f_{K_{f}}}^{-1}\left(C_{2}\right) \oplus E_{f_{K_{f}}}^{-1}\left(C_{4}\right)=\delta$.

8. Repeat Steps $2-7$ until a subkey is suggested 4 times.

We call Steps 2-5 an $\epsilon$-shift as each plaintext is encrypted, then shifted by all possible $\epsilon$ 's and the values of the shifted ciphertexts are decrypted.

The time complexity of Step 1 is equivalent to $2^{m_{b}+m_{f}}$ memory accesses. However, we can keep the counters in a more efficient data structures (like Btrees, or dynamic hash tables), for which Step 1 takes only one memory access.

Each $F$ induces a set of $2^{r_{b}}$ ciphertexts in $G$, and each ciphertext is shifted by $2^{t_{f}}$ possible values, hence $|H|=|I|=2^{r_{b}+t_{f}}$. Even though we expand the number of possible quartets (by multiplying the size of $I$ by $2^{t_{f}}$ ), the number of right quartets does not change. Hence, we still need about $\left\lceil 8(\hat{p} \hat{q})^{-2} / 2^{r_{b}+1}\right\rceil$ structures. 
The data complexity of the attack is $N=2^{r_{b}+t_{f}} \cdot\left\lceil 8(\hat{p} \hat{q})^{-2} / 2^{r_{b}+1}\right\rceil$. However, we might get that $N>2^{n}$. We can implement these cases in one of two ways. The first way is to ask for the encryption/decryption $N$ (not necessarily different) oracle queries. The second way to implement this is to store the already encrypted/decrypted values in a table, and test for each encryption/decryption if it is already in the table, in order to save most of the encryptions/decryptions. This way the attack requires $2^{n}$ known plaintexts and $N$ memory accesses.

Like in the previous section we perform the time complexity analysis for each $F$ independently of other $F$ 's, as each execution of Steps 6-7 for a given structure is independent of the execution of these steps for some other structure.

Like in the previous section, Step 6 inserts into a hash table the plaintexts. Thus, the time complexity of Step 6 is $2^{r_{b}+t_{f}}$ memory accesses per structure, and $N$ memory accesses in total.

Since each collision in the hash table of Step 6 suggests a quartet, we have in this step about $2^{3 r_{b}+2 t_{f}-n-1}$ memory accesses for each structure $F$, and the expected number of remaining quartets is $2^{3 r_{b}+2 t_{f}-n-1} \cdot\left(2^{t_{b}-r_{b}}\right)^{2}=$ $2^{2 t_{b}+r_{b}+2 t_{f}-n-1}$. Since there are $N / 2^{r_{b}+t_{f}}$ structures we conclude that for the whole attack this step requires about $N \cdot 2^{2 r_{b}+t_{f}-n-1}$ memory accesses.

We recover subkey material both in $E_{b}$ and $E_{f}$. By repeating the analysis from Section 3 each remaining quartet suggests $2^{m_{b}+m_{f}-2 t_{b}-2 t_{f}-2}$ subkeys for $E_{b}$ and $E_{f}$, and thus, we get $2^{r_{b}+m_{b}+m_{f}-n-3}$ hits (on average) from each structure and $N \cdot 2^{m_{b}+m_{f}-n-t_{f}-3}$ subkey hits in total. We conclude that Step 7(b) requires $N \cdot 2^{t_{b}+t_{f}-n-1} \cdot\left(2^{m_{b}+t_{f}}+2^{m_{f}+t_{b}}\right)$ memory accesses for the whole attack.

As there are about $N \cdot 2^{m_{b}+m_{f}-t_{f}-n-3}$ subkey hits in total, the expected number of hits per subkey is about $N \cdot 2^{-t_{f}-n-3}$. Note that $N$ might be bigger than $2^{n}$ but on the same time, $N \leq 2^{n+t_{f}}$ (as we take at most $2^{n}$ ciphertexts and shift them by $2^{t_{f}}$ values). We again find that the number of hits per wrong subkey is $\leq 1 / 8$.

In Step 8 we check whether one of the counters has the value of 4 (or more). Using the same methods as in the previous section, we can use more appropriate data structures which reduce the time complexity of this step to 1 memory accesses for each $F$, and for the entire attack $N / 2^{r_{b}+t_{f}}$ memory accesses.

The data complexity of the attack is $N=2^{r_{b}+t_{f}} \cdot\left\lceil 8(\hat{p} \hat{q})^{2} / 2^{r_{b}+1}\right\rceil$ adaptive chosen plaintexts and ciphertexts (and as stated earlier if $N>2^{n}$ we can replace it by $2^{n}$ known plaintexts using a table of size $2^{n}$ ). The expected time complexity of the attack is $N\left(2+2^{2 r_{b}+t_{f}-n-1}+2^{m_{b}+t_{b}+2 t_{f}-n-1}+2^{m_{f}+2 t_{b}+t_{f}-n-1}\right)$ memory accesses. The memory complexity of the attack is $2^{m_{b}+m_{f}}+2^{r_{b}+t_{f}}$.

Table 3 summarizes the time complexity of each step and the number of plaintexts / pairs / quartets that remain after each step of the algorithm.

We use the same decomposition of 3.5-round SC2000 as in Section 3 For this decomposition the following properties were presented in [8]: $r_{b}=r_{f}=m_{b}=$ $m_{f}=40, t_{b}=27, t_{f}=27.9, n=128, \hat{p}=2^{-8.96}, \hat{q}=2^{-9.16}$. Hence, the data complexity of the attack is $2^{67.9}$ adaptive chosen plaintexts and ciphertexts (this complexity can be reduced to $2^{67}$ by attacking $E^{-1}$ ). The attack requires $2^{67.9}$ memory cells (when we attack $E^{-1}$ it requires only $2^{67}$ memory cells). The time 
Table 3. The Steps of the Improved Boomerang Attack and Their Effect

\begin{tabular}{llll}
\hline Step Short & Time & \# Remaining Texts/ \\
No. & Description & Complexity & Pairs/ Quartets \\
\hline 1 & Subkey counters' init. & $1 \mathrm{MA}$ & - \\
$2+3$ & Data generation & $N / 2^{t_{f}}$ encryptions & $N / 2^{t_{f}}$ plaintexts \\
4 & Data generation & $N \mathrm{MA}$ & No change \\
5 & Data generation & $N$ decryptions & $N$ Plaintexts \\
6 & Eliminating wrong pairs & $N \mathrm{MA}$ & $N 2^{2 r_{b}+2 t_{f}-n-2}$ qts. \\
$7($ a) & Eliminating quartets & $N 2^{2 r_{b}+t_{f}-n-1} \mathrm{MA}$ & $N 2^{2 t_{b}+2 t_{f}-n-2}$ qts. \\
$7(\mathrm{~b})$ & Subkey detection & $N 2^{t_{b}+t_{f}-n-2}\left(2^{m_{b}+t_{f}}+2^{m_{f}+t_{b}}\right) \mathrm{MA}$ No change \\
8 & Printing subkey & $N / 2^{r_{b}+t_{f}} \mathrm{MA}$ & No change \\
\hline
\end{tabular}

\section{MA - Memory Accesses}

complexity of the attack is $2^{68.9}$ memory accesses (the attack on $E^{-1}$ requires $2^{68}$ memory accesses).

We also use the same decomposition of 10-round Serpent like in Section 3 . For this decomposition the following properties were presented in [7]: $r_{b}=m_{b}=$ $76, r_{f}=m_{f}=20, t_{b}=48.85, t_{f}=13.6, n=128, \hat{p}=2^{-25.4}, \hat{q}=2^{-34.9}$. The data complexity of the attack is $N=2^{123.6+13.6}=2^{137.2}$. As mentioned before, we can either treat this as $2^{137.2}$ queries to the encryption/decryption oracle (of course not distinct queries) or we can just ask the encryption/decryption of any plaintext/ciphertext we need, and store it in a table. The attack requires $2^{173.8}$ memory accesses.

Table 4. Comparison of the Boomerang and the Rectangle Generic Attacks

\begin{tabular}{|c|c|c|c|}
\hline$\overline{\text { Attack }}$ & $\begin{array}{l}\text { Rectangle } \\
\text { (Section 3) }\end{array}$ & $\begin{array}{l}\text { Boomerang } \\
\text { (Section 4) }\end{array}$ & $\begin{array}{l}\text { Enhanced } \\
\text { Boomerang (Section } 5\end{array}$ \\
\hline $\begin{array}{l}\text { Cipher's parts } \\
\text { being attacked }\end{array}$ & $E_{f} \circ E_{1} \circ E_{0} \circ E_{b}$ & $E_{1} \circ E_{0} \circ E_{b}$ & $E_{f} \circ E_{1} \circ E_{0} \circ E_{b}$ \\
\hline $\begin{array}{l}\text { Type of } \\
\text { Attack }\end{array}$ & Chosen Plaintext & $\begin{array}{l}\text { Adaptive Chosen } \\
\text { Plaintext and Ciphert. }\end{array}$ & $\begin{array}{l}\text { Adaptive Chosen } \\
\text { Plaint. and Ciphertext }\end{array}$ \\
\hline $\begin{array}{l}\text { Data } \\
\text { Complexity }(\mathrm{N})\end{array}$ & $\max \left\{2^{r_{b}}, 2^{n / 2+2} / \hat{p} \hat{q}\right\}$ & $\max \left\{8(\hat{p} \hat{q})^{-2}, 2^{r_{b}}\right\}$ & $2^{t_{f}} \max \left\{2^{r_{b}}, 8(\hat{p} \hat{q})^{-2}\right\}$ \\
\hline $\begin{array}{l}\text { Memory } \\
\text { Accesses }\end{array}$ & $\begin{array}{l}N^{2}\left(2^{r_{f}-n-1}+2^{t_{f}-n}\right. \\
+2^{2 t_{f}+2 r_{b}-2 n-2} \\
+2^{m_{b}+2 t_{f}+t_{b}-2 n-1} \\
\left.+2^{m_{f}+2 t_{b}+t_{f}-2 n-1}\right) \\
+N\end{array}$ & $\begin{array}{l}N\left(1+2^{2 r_{b}-n-2}+\right. \\
\left.2^{t_{b}+m_{b}-n-1}\right)\end{array}$ & $\begin{array}{l}N\left(2+2^{2 r_{b}+t_{f}-n-1}+\right. \\
2^{m_{b}+t_{b}+2 t_{f}-n-1}+ \\
\left.2^{m_{f}+t_{f}+2 t_{b}-n-1}\right)\end{array}$ \\
\hline Memory Cells & $2^{m_{b}+m_{f}}+N$ & $2^{r_{b}}+2^{m_{b}}$ & $2^{r_{b}+t_{f}}+2^{m_{b}+m_{f}}$ \\
\hline Subkey Bits & $m_{b}+m_{f}$ & $m_{b}$ & $m_{b}+m_{f}$ \\
\hline $\begin{array}{l}\text { Hits per } \\
\text { Wrong Subkey }\end{array}$ & $\leq 1 / 16$ & $\leq 1 / 8$ & $\leq 1 / 8$ \\
\hline
\end{tabular}


Table 5. New Boomerang and Rectangle Results on SC2000 and Serpent

\begin{tabular}{lllccc}
\hline Cipher & Attack & Number & \multicolumn{3}{c}{ Complexity } \\
\cline { 3 - 6 } & & of Rounds & Data & Time & Memory \\
\hline SC2000 Rectangle - this paper & 3.5 & $2^{84.6} \mathrm{CP}$ & $2^{84.6} \mathrm{MA}$ & $2^{84.6}$ \\
& Boomerang - this paper 3 & $2^{41} \mathrm{ACPC}$ & $2^{41} \mathrm{MA}$ & $2^{40}$ \\
& Boomerang - this paper 3.5 & $2^{67} \mathrm{ACPC}$ & $2^{67} \mathrm{MA}$ & $2^{67}$ \\
best & 4.5 & $2^{104.3} \mathrm{KP}$ & $2^{83.3} \mathrm{MA}$ & $2^{80}$ \\
\hline Serpent Amp. Boomerang[10] & 9 & $2^{110} \mathrm{CP}$ & $2^{252} \mathrm{MA}$ & $2^{208}$ \\
& Rectangle[7] & 10 & $2^{126.8} \mathrm{CP}$ & $2^{217} \mathrm{MA}$ & $2^{192}$ \\
& Rectangle[7] & 10 & $2^{126.8} \mathrm{CP}$ & $2^{219.4} \mathrm{MA}$ & $2^{126.8}$ \\
& Boomerang - this paper 9 & $2^{123.6} \mathrm{ACPC}$ & $2^{123.6} \mathrm{MA}$ & $2^{21.5}$ \\
& Boomerang - this paper 10 & $2^{128} \mathrm{KP}$ & $2^{173.8} \mathrm{MA}$ & $2^{96}$ \\
best & Rectangle - this paper & 10 & $2^{126.3} \mathrm{CP}$ & $2^{173.8} \mathrm{MA}$ & $2^{126.3}$ \\
\hline MA - Memory Accesses & 11 & $2^{118} \mathrm{KP}$ & $2^{214} \mathrm{MA}$ & $2^{85}$ \\
CP - Chosen Plaintexts, KP - Known Plaintexts & & & \\
ACPC - Adaptive Chosen Plaintexts and Ciphertexts & & &
\end{tabular}

\section{Summary}

This paper presents several contributions. The first contribution is an improved generic rectangle attack. The improved attack algorithm can attack 10-round Serpent with data complexity of $2^{126.3}$ chosen plaintexts and time complexity of $2^{173.8}$ memory accesses. This new result enables attacking 10-round Serpent with 192-bit subkeys. We also have shown that the algorithm is very successful and almost always reduces the number of candidate subkeys.

The second contribution is a generic boomerang key recovery attack. The attack uses similar techniques as in the rectangle key recovery attack and the result is an efficient algorithm for retrieving subkey material. In the analysis of this attack we found out that this attack also almost always succeeds.

The third contribution is extending the generic boomerang key recovery attack to attack more rounds. This contribution allows for the boomerang attack to attack as many rounds as the rectangle attack despite its adaptive chosen plaintext and ciphertext nature. This allows to attack 10-round Serpent with the enhanced boomerang attack using $2^{137.2}$ adaptive chosen plaintexts and ciphertexts (or $2^{128}$ known plaintexts), and $2^{173.8}$ memory accesses.

In Table 4 we compare the requirements of the generic attacks, and in Table 5 we present our new results on Serpent and SC2000. For comparison, we also include the previous boomerang and rectangle results and the best known attacks against these ciphers.

\section{References}

1. Ross Anderson, Eli Biham, Lars R. Knudsen, Serpent: A Proposal for the Advanced Encryption Standard, NIST AES Proposal, 1998. 
2. Eli Biham, Higher Order Differential Cryptanalysis, unpublished paper, 1994.

3. Eli Biham, Adi Shamir, Differential Cryptanalysis of the Data Encryption Standard, Springer-Verlag, 1993.

4. Eli Biham, Alex Biryukov, Adi Shamir, Cryptanalysis of Skipjack reduced to 31 rounds, Advances in Cryptology, proceedings of EUROCRYPT '99, LNCS 1592, pp. 12-23, Springer-Verlag, 1999.

5. Eli Biham, Alex Biryukov, Adi Shamir, Miss in the Middle Attacks on IDEA and Khufu, proceedings of Fast Software Encryption 6, LNCS 1636, pp. 124-138, Springer-Verlag, 1999.

6. Eli Biham, Orr Dunkelman, Nathan Keller, Linear Cryptanalysis of Reduced Round Serpent, proceedings of Fast Software Encryption 8, 2001, to appear.

7. Eli Biham, Orr Dunkelman, Nathan Keller, The Rectangle Attack-Rectangling the Serpent, Advances in Cryptology, proceedings of EUROCRYPT '01, LNCS 2045, pp. 340-357, Springer-Verlag, 2001.

8. Orr Dunkelman, Nathan Keller, Boomerang and Rectangle Attacks on SC2000, preproceedings of the NESSIE second workshop, 2001.

9. Louis Granboulan, Flaws in Differential Cryptanalysis of Skipjack, proceedings of Fast Software Encryption 8, 2001, to appear.

10. John Kelsey, Tadayoshi Kohno, Bruce Schneier, Amplified Boomerang Attacks Against Reduced-Round MARS and Serpent, proceedings of Fast Software Encryption 7, LNCS 1978, pp. 75-93, Springer-Verlag, 1999.

11. Lars Knudsen, Truncated and Higher Order Differentials, proceedings of Fast Software Encryption 2, LNCS 1008, pp. 196-211, Springer-Verlag, 1995.

12. Lars Knudsen, Håvard Raddum, A Differential Attack on Reduced-Round SC2000, preproceedings of the NESSIE second workshop, 2001.

13. Lars Knudsen, Matt J.B. Robshaw, David Wagner, Truncated Differentials and Skipjack, Advances in Cryptology, proceedings of CRYPTO '99, LNCS 1666, pp. 165-180, Springer-Verlag, 1999.

14. Suzan K. Langford, Martin E. Hellman, Differential-Linear Cryptanalysis, Advances in Cryptology, proceedings of CRYPTO '94, LNCS 839, pp. 17-25, SpringerVerlag, 1994.

15. Mitsuru Matsui, Linear Cryptanalysis Method for DES Cipher, Advances in Cryptology, proceedings of EUROCRYPT '93, LNCS 765, pp. 386-397, Springer-Verlag, 1994.

16. NESSIE - New European Schemes for Signatures, Integrity and Encryption. http://www.nessie.eu.org/nessie/.

17. Takeshi Shimoyama, Hitoshi Yanami, Kazuhiro Yokoyama, Masahiko Takenaka, Kouichi Itoh, Jun Yajima, Naoya Torii, Hidema Tanaka, The Block Cipher SC2000, proceedings of Fast Software Encryption 8, 2001, to appear.

18. David Wagner, The Boomerang Attack, proceedings of Fast Software Encryption 6, LNCS 1636, pp. 156-170, Springer-Verlag, 1999.

19. Hitoshi Yanami, Takeshi Shimoyama, Orr Dunkelman, Differential and Linear Cryptanalysis of a Reduced-Round SC2000, these proceedings. 